\title{
Road Curb Tracking in an Urban Environment
}

\author{
Wijesoma W. S., Kodagoda K. R. S., Balasuriya A. P. \\ School of Electrical and Electronic Engineering \\ Nanyang Technological University \\ Singapore \\ \{eswwijesoma, ps6983882j, earjuna\}@ntu.edu.sg
}

\author{
Challa S. \\ Dept. of Electrical and Electronic Engineering \\ The University of Melbourne \\ Australia \\ s.challa@ee.mu.oz.au
}

\begin{abstract}
Road detection and tracking is very useful in the synthesis of driver assistance and intelligent transportation systems. In this paper a methodology is proposed based on the extended Kalman filer for robust road curb detection and tracking using a combination of onboard active and passive sensors. The problem is formulated as detecting and tracking a maneuvering target in clutter using onboard sensors on a moving platform. The primary sensors utilized are a 2 dimensional SICK laser scanner, five encoders and a gyroscope, together with an image sensor (CCD camera). Compared to the active $2 D$ laser scanner the CCD camera is capable of providing observations over an extended horizon, thus making available much useful information about the curb trend, which is exploited in mainly the laser based tracking algorithm. The advantage of the proposed image enhanced laser detection/tracking method, over laser alone detection/tracking, is illustrated using simulations and its robustness to varied road curvatures, branching, turns and scenarios, is demonstrated through experimental results.
\end{abstract}

Keywords: Tracking, filtering, estimation, fusion.

\section{Introduction}

The detection and tracking of road boundaries is crucial in intelligent vehicle applications. Road boundaries can be used for autonomous driving, and driver assistance systems, such as road departure or lane excursion warning systems and forward collision warning systems. In this paper the concern is about detecting and tracking of road curbs. Road curbs are prevalent in most urban, semiurban, road environments and places such as theme parks, industrial estates and campus sites. In Singapore road curbs exists in every urban, suburban and downtown area and is the main inspiration and motivation for the work described. Our approach is thus to detect and track curbs with the objective eventually using such information as an aid for driver assistance and autonomous driving.

There are a number of papers in the literature that address the road boundary detection problem and they can be broadly categorized by the choice of sensors utilized. Methodologies that use only CCD cameras are the most extensively researched and tested [1-3]. Reasons for the camera's success as an automotive road sensor are: the rich information content, lower costs, lower operating power and absence of sweep time. However, shadows, poor lighting and bad weather conditions are a few major factors that can adversely impair techniques based on solely vision. Millimeter wave radar (MMWR) [4-6] and laser measurement systems (LMS) [7-8] being active sensing techniques overcome the limitations to a large extent in road sensing. More specifically MMWR has the advantage of operating over long distances $(120 \mathrm{~m})$ in snowy, hazy, dusty and rainy environments without affecting quality of detection, and versions of laser range/bearing sensors operate over moderate distances $(1 \mathrm{~m}-80 \mathrm{~m})$ and can operate under moderately bad weather conditions, although they are not usable under extremely harsh conditions. It is meaningful to consider fusing both the passive vision and active laser/radar modalities to improve the robustness of road detection. For example in [9] a MMWR and a CCD camera are used for road detection. Although the MMWR has the above-mentioned advantages, cost, operating power, signal clutter and size considerations can tilt the balance in favor of LMS for automotive applications. Thus, in our work, a 2 dimensional laser measurement system (see Figure 1) and a CCD camera are used for road curb and midline detection and tracking. Here the strong correlation that exists between road curbs and lane marking are exploited in the detection/tracking algorithm.

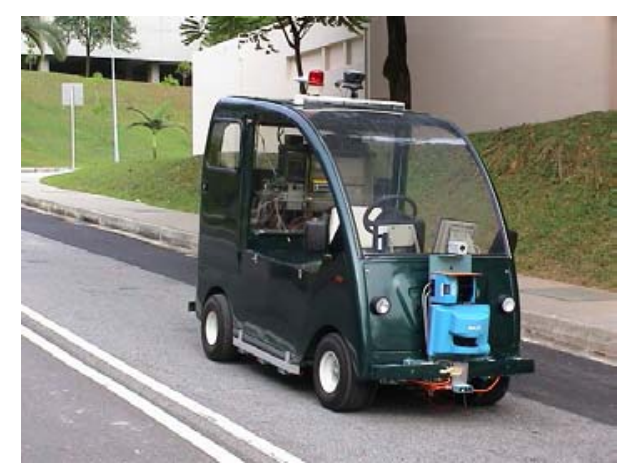

Figure 1. Experimental vehicle (GenOME) with sensors

In Section 2 the problem of detecting and tracking road curbs is formulated. Section 3 develops a Kalman filterbased laser alone tracking system, while in Section 4 an 
image-enhanced tracking algorithm is developed. In section 5 simulation results are presented and in section 6 some experimental results are presented and discussed. Section 7 concludes the paper.

\section{Problem Formulation}

In this section we begin with the formulation of the problem of road feature detection and tracking.

Coordinate Frames: There are three coordinate frames of interest. A fixed world coordinate frame $\left\{X_{w}, Y_{w}\right\}$ used mainly for visualization purposes, and two coordinate frames $\left\{{ }^{k} X_{v},{ }^{k} Y_{v}\right\}$ and $\left\{{ }^{k+1} X_{v},{ }^{k+1} Y_{v}\right\}$ denoting the vehicle frame at the time instances $k$ and $k+1$ respectively (see Figure 2) for derivation of the curb tracking algorithm. The relationship between the vehicle frames at the $k+1^{\text {th }}$ instant and the $k^{\text {th }}$ instant is a simple rotation $\left(\Delta \phi_{v}\right)$ followed by a translation $\left(t_{x}, t_{y}\right)$ as shown in Figure 2.

Target Model: Since the objective is to track curbs (left/right) using the vehicle mounted ladar scanner, a pseudo target(s) is defined as the line segment that results from the intersection of the planar ladar scan with the planar (left/right) curb surface(s). Thus, when the vehicle is in motion the line segment(s) or target(s) moves along the curb (left/right). A line is represented by a point $(x, y)$ and its orientation $(\phi)$. Thus, the state vector, $X$, is defined as $X \in\left\{\left({ }^{k} x^{R},{ }^{k} y^{R},{ }^{k} \phi^{R}\right),\left({ }^{k} x^{L},{ }^{k} y^{L},{ }^{k} \phi^{L}\right)\right\}$, where $\left({ }^{k} x^{R},{ }^{k} y^{R},{ }^{k} \phi^{R}\right)$ and $\left({ }^{k} x^{L},{ }^{k} y^{L},{ }^{k} \phi^{L}\right)$ denote the right and left curb segments with respect to the $k^{\text {th }}$ vehicle frame. Since the road is locally flat, the target (laser scan and curb intersection) would be approximately at a known distance $\bar{d}$ in the direction of the vehicle $y$-axis. From Figure 2, it may be easily deduced that the evolution of the curb segments is described the by equation (1).

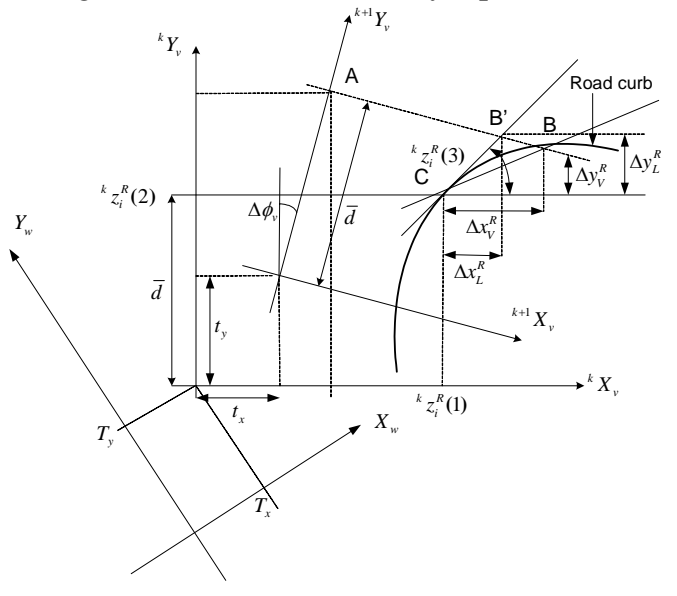

Figure 2. Road curb and coordinate frames
The target model is,

$$
{ }^{k+1} \mathbf{x}(k+1)=\mathbf{f}\left[{ }^{k} \mathbf{x}(k),{ }^{k} \mathbf{u}(k)\right]+\mathbf{v}(k)
$$

where,

$$
\begin{aligned}
& { }^{k} \mathbf{x}(k)=\left[{ }^{k} x^{R}(k),{ }^{k} y^{R}(k),{ }^{k} \phi^{R}(k),{ }^{k} x^{L}(k),{ }^{k} y^{L}(k),{ }^{k} \phi^{L}(k)\right]^{T} \\
& \mathbf{f}\left[{ }^{k} \mathbf{x}(k),{ }^{k} \mathbf{u}(k)\right]=\mathbf{F}(k){ }^{k} \mathbf{x}(k)+{ }^{k} \mathbf{u}(k), \\
& \mathbf{F}(k)=\left[\begin{array}{cc}
\mathbf{R}_{v} & \mathbf{O}_{3 x 3} \\
\mathbf{O}_{3 x 3} & \mathbf{R}_{v}
\end{array}\right], \mathbf{R}_{v}=\left[\begin{array}{ccc}
\cos \Delta \phi_{v} & -\sin \Delta \phi_{v} & 0 \\
\sin \Delta \phi_{v} & \cos \Delta \phi_{v} & 0 \\
0 & 0 & 1
\end{array}\right], \\
& { }^{k} \mathbf{u}(k)=\left[\begin{array}{l}
\left(\Delta x_{j}^{R}-t_{x}\right) \cos \Delta \phi_{v}-\left(\Delta y_{j}^{R}-t_{y}\right) \sin \Delta \phi_{v} \\
\left(\Delta x_{j}^{R}-t_{x}\right) \sin \Delta \phi_{v}+\left(\Delta y_{j}^{R}-t_{y}\right) \cos \Delta \phi_{v} \\
\Delta \phi_{j}^{R}-\Delta \phi_{v} \\
\left(\Delta x_{j}^{L}-t_{x}\right) \cos \Delta \phi_{v}-\left(\Delta y_{j}^{L}-t_{y}\right) \sin \Delta \phi_{v} \\
\left(\Delta x_{j}^{L}-t_{x}\right) \sin \Delta \phi_{v}+\left(\Delta y_{j}^{L}-t_{y}\right) \cos \Delta \phi_{v} \\
\Delta \phi_{j}^{L}-\Delta \phi_{v}
\end{array}\right] ; j=L, V
\end{aligned}
$$

$j=L, V$ means either Laser or Vision. $\mathbf{v}(k)$ is the zeromean white Gaussian process noise. $E\left[\mathbf{v}(k) \mathbf{v}(k)^{T}\right]=\mathbf{Q}(k)$. All other symbols as per defined in the figure 2.

Observation model: The group observation model is considered consisting of laser as well as vision data and hence it is a $12 \times 1$ vector.

${ }^{k+1} \mathbf{z}(k)=\mathbf{h}\left[{ }^{k+1} \mathbf{x}(k)\right]+\mathbf{w}(k)$

where, $\quad{ }^{k+1} \mathbf{z}(k)=\left[\mathbf{z}_{L}^{R}, \mathbf{z}_{L}^{L}, \mathbf{z}_{V}^{R}, \mathbf{z}_{V}^{L}\right]^{T}$, In $\mathbf{z}_{L}^{R}$, subscript $L$ means Laser and superscript $R$ means Right side and also $\quad \mathbf{z}_{L}^{R}=\left[z_{x}, z_{y}, z_{\phi}\right]^{T} . \quad \mathbf{h}\left[{ }^{k+1} \mathbf{x}(k)\right]=\left[\begin{array}{l}\mathbf{H}_{L} \\ \mathbf{H}_{V}\end{array}\right]{ }^{k+1} \mathbf{x}(k)$, where, $\mathbf{H}_{L}=\mathbf{H}_{V}=\mathbf{I}_{6 \times 6} . \mathbf{w}(k)$ is the sequence of zeromean white Gaussian measurement noise. $E\left[\mathbf{w}(k) \mathbf{w}(k)^{T}\right]=\mathbf{R}(k)$ is a block diagonal matrix.

Aim: Our aim is to compute the estimates of $\mathbf{x}$ given the observations, $\mathbf{z}$, in an optimal and robust manner.

\section{Filter prediction:}

$$
\begin{aligned}
& { }^{k+1} \mathbf{x}(k+1 / k)=\mathbf{f}\left[{ }^{k} \mathbf{x}(k / k),{ }^{k} \mathbf{u}(k)\right] \\
& \mathbf{P}(k+1 / k)=\left(\frac{\partial \mathbf{f}}{\partial \mathbf{x}}\right) \mathbf{P}(k / k)\left(\frac{\partial \mathbf{f}}{\partial \mathbf{x}}\right)^{T}+\left(\frac{\partial \mathbf{f}}{\partial \mathbf{u}}\right) \boldsymbol{\Lambda}_{\mathbf{u}}\left(\frac{\partial \mathbf{f}}{\partial \mathbf{u}}\right)^{T}+\mathbf{Q}
\end{aligned}
$$

where, $\boldsymbol{\Lambda}_{\mathbf{u}}$ is the covariance matrix of $\mathbf{u}$. 
Filter update:

$$
\begin{aligned}
&{ }^{k+1} \hat{\mathbf{x}}(k+1 / k+1)={ }^{k+1} \hat{\mathbf{x}}(k+1 / k) \\
&+\mathbf{R}_{x z} \mathbf{R}_{z z}^{-1}\left({ }^{k+1} \mathbf{z}(k+1)-\mathbf{h}\left[{ }^{k+1} \hat{\mathbf{x}}(k+1 / k)\right]\right) \\
& \mathbf{P}(k+1 / k+1)=\mathbf{R}_{x x}-\mathbf{R}_{x z} \mathbf{R}_{z z}^{-1} \mathbf{R}_{z x}
\end{aligned}
$$

where,

$$
\begin{aligned}
& \mathbf{R}_{x x}=\mathbf{P}(k+1 / k), \mathbf{R}_{z z}=\left(\frac{\partial \mathbf{h}}{\partial \mathbf{x}}\right) \mathbf{P}(k+1 / k)\left(\frac{\partial \mathbf{h}}{\partial \mathbf{x}}\right)^{T}+\mathbf{R} \\
& \mathbf{R}_{x z}=\mathbf{P}(k+1 / k)\left(\frac{\partial \mathbf{h}}{\partial \mathbf{x}}\right)^{T}, \mathbf{R}_{z x}=\left(\frac{\partial \mathbf{h}}{\partial \mathbf{x}}\right) \mathbf{P}(k+1 / k)
\end{aligned}
$$

\section{Laser Alone Road Curb Tracking}

In this section we formulate the road curb-tracking problem only using the laser data (road curbs [11]). Structure of Equation (1) and (2) remain the same, however, the parameters can be different. The parameters of $\mathbf{u}(k)$ in equation (1) can be calculated as (see Figure 2),

$$
\begin{aligned}
& \Delta x_{L}^{i}=\frac{\left(c_{B^{\prime} C}^{i}-c_{A B^{\prime}}\right)}{\left(m_{A B^{\prime}}-m_{B^{\prime} C}^{i}\right)}-{ }^{k} \mathbf{z}_{L}^{i}(1) \\
& \Delta y_{L}^{i}=m_{A B^{\prime}}\left(\frac{\left(c_{B^{\prime} C}^{i}-c_{A B^{\prime}}\right)}{\left(m_{A B^{\prime}}-m_{B^{\prime} C}^{i}\right)}+c_{A B^{\prime}}-{ }^{k} \mathbf{z}_{L}^{i}(2)\right. \\
& \Delta \phi_{L}^{i}=0 \\
& m_{A B^{\prime}}=-\tan \Delta \phi_{v} \\
& c_{A B^{\prime}}=t_{y}+\bar{d} \cos \Delta \phi_{v}+\tan \Delta \phi_{v}\left(t_{x}+\bar{d} \sin \Delta \phi_{v}\right) \\
& m_{B^{\prime} C}=\tan { }^{k} \mathbf{z}_{L}^{i}(3) \\
& c_{B^{\prime} C}={ }^{k} \mathbf{z}_{L}^{i}(2)-m_{B^{\prime} C}{ }^{k} \mathbf{z}_{L}^{i}(1)
\end{aligned}
$$

where, $i=L, R$; meaning Left or Right.

Each line is modeled as $y=m x+c . m_{B^{\prime} C}^{R}$ means the line going through $B^{\prime} C$ corresponding to the right side of the vehicle. In equation (2), $\mathbf{z}(k)$ only consists of laser data (therefore, a $6 \times 1$ vector), $\mathbf{H}=\mathbf{I}_{6 \times 6}$ and $\mathbf{R}$ is a $6 \times 6$ matrix.

Data association: Due to the presence of clutter, a data association step has to be carried out and in this case we pick the measurement with the smallest normalized innovation as a single candidate measurement of the target. If there is no candidate measurement lying inside the validation gate (using the Chi-square distribution), no measurement is considered and the filter simply does the prediction without update.

\section{Image Enhanced Curb Tracking}

In this method, we utilize the extracted image features (road boundaries [12]) with laser data (road curbs [11]) in curb tracking. We use the target model and the measurement model defined by equations (1) and (2), however with the following parameter values (see Figure 2.).

$$
\begin{aligned}
& \Delta x^{i}=\frac{\left(c_{B C}^{i}-c_{A B}\right)}{\left(m_{A B}-m_{B C}^{i}\right)}-\mathbf{z}_{V}^{i}(1) \\
& \Delta y^{i}=m_{A B} \frac{\left(c_{B C}^{i}-c_{A B}\right)}{\left(m_{A B}-m_{B C}^{i}\right)}+c_{A B}-\mathbf{z}_{V}^{i}(2) \\
& \Delta \phi^{i}=\tan ^{-1} m_{B C}^{i}-\mathbf{z}_{V}^{i}(3) \\
& m_{A B}=-\tan \Delta \phi_{v} \\
& c_{A B}=t_{y}+\bar{d} \cos \Delta \phi_{v}+\tan \Delta \phi_{v}\left(t_{x}+\bar{d} \sin \Delta \phi_{v}\right)
\end{aligned}
$$

where, $i=L, R$; meaning Left or Right. $m_{B C}^{i}$ and $c_{B C}^{i}$ values are obtained from the fitted lines for the vision data along the curb segment $B C$. It is to be noted that it can be made more accurate by fitting a polynomial to the vision data of the segment BC. However, due to the limitation of the maximum road curvature that can have, it is reasonable to use a straight line fit rather than a more complex polynomial fit.

Data association: Data association using the smallest normalized innovation of the tracking filter will fail particularly in road bends due to the lack of the information about the future road curvatures. Methods exist that can handle such situations (eg. PDAF-IMMs for tracking maneuvering targets in clutter[10]), at the expense of increasing complexity and computational requirements. One of the challenging problems in our case is that the laser may provide cluttered measurements with or without a measurement corresponding to the actual target. The image sensor can be effectively used to overcome those problems.

Let us assume the measurements of laser data (or vision data) in vehicle coordinates are having the same uncertainties, $\sigma_{j}^{2} ; j=L, V$, where $L=$ laser and $V=$ vision data respectively. Now, we can calculate the variance of the best-fitted line parameters as follows.

Using the maximum likelihood, in the case of Gaussian distributed uncertainties, the best fit for parameters, $\mathbf{a}=\left\{a_{k}\right\}$ with measurement, $y=f(\mathbf{x}, \mathbf{a})$ is the one that minimizes the $\chi^{2}$ function,

$\chi^{2}=\sum_{i} \frac{\left(y_{i}-f\left(\mathbf{x}_{i}, \mathbf{a}\right)\right)^{2}}{\sigma_{i}^{2}}$ 
where, $\sigma_{i}^{2}$ is the variance of data. The information matrix, $\mathbf{H}^{I}$ can be defined as,

$H_{j k}^{I}=\frac{1}{2} \frac{\partial^{2} \chi^{2}}{\partial a_{j} \partial a_{k}}$

Then, variances and covariances of $\mathbf{a}=\left\{a_{k}\right\}$ are given by,

$V_{a_{j}, a_{k}}=\left(H_{j k}^{I}\right)^{-1}$

If the line is represented by, $y=\tan \phi_{l} x+c_{l}$, the perpendicular distance from a point $\left(x_{i}, y_{i}\right)$ to the line is given by,

$d_{i}=x_{i} \sin \phi_{l}-y_{i} \cos \phi_{l}+c_{l} \cos \phi_{l}$

Finding the best-fit parameters, which minimizes $d_{i}$ in equation (8) can be formulated as a minimization of $\chi^{2}$

as, $\chi^{2}\left(\phi_{l}, c_{l}\right)=\frac{1}{\sigma_{l}^{2}} \sum_{i}\left(x_{i} \sin \phi_{l}-y_{i} \cos \phi_{l}+c_{l} \cos \phi_{l}\right)^{2}$

and therefore, we can calculate variance of the angle of the fitted line, $\sigma_{\phi_{j}}^{2} ; j=L, V$. We assume the variance of a point on the fitted line is as the same as the variance of the data itself. Let the line parameters at time $k$ is $\psi_{k}=\left[x_{l}, y_{l}, \phi_{l}\right]^{T}$ and its covariance matrix is,

$$
\Lambda_{k}^{\psi}=\left(\begin{array}{ccc}
\sigma_{x l}^{2} & 0 & 0 \\
0 & \sigma_{y l}^{2} & 0 \\
0 & 0 & \sigma_{\phi l}^{2}
\end{array}\right)
$$

Lets consider the transformation of line data from $k^{\text {th }}$ frame $\psi_{k}=\left[x_{l}, y_{l}, \phi_{l}\right]^{T}$ to $k+l^{\text {th }}$ frame, $\psi_{k+1}$, (see Figure 2),

$\hat{\psi}_{k+1}=\mathbf{R}_{v} \psi_{k}+\mathbf{u}$

and propagation of uncertainties,

$$
\Lambda_{k+1}^{\hat{\psi}}=\mathbf{R}_{v} \Lambda_{k}^{\psi} \mathbf{R}_{v}^{T}+\left(\frac{\partial \mathbf{R}_{v}}{\partial \Delta \phi_{v}}\right) \Lambda_{\mathbf{R}_{v}}\left(\frac{\partial \mathbf{R}_{v}}{\partial \Delta \phi_{v}}\right)^{T}+\Lambda_{\mathbf{u}}
$$

where, $\Lambda_{\mathbf{R}_{v}}$ and $\Lambda_{\mathbf{u}}$ are uncertainties in rotation and translation respectively. The Mahalanobis distances are calculated for all the candidate laser line segments in $k+I^{\text {th }}$ time instant with the transformed vision line data from the $k^{\text {th }}$ time instant to $k+l^{\text {th }}$ time instant as follows.
$d_{m}=\left(\hat{\psi}_{k+1}^{V}-\psi_{k+1}^{L}\right)^{T}\left(\Lambda_{k+1}^{\hat{\psi}}+\Lambda_{k}^{\psi}\right)^{-1}\left(\hat{\psi}_{k+1}^{V}-\psi_{k+1}^{L}\right)$

For the values of $d_{m}$, which are less than a threshold $\kappa$, (determined using $\chi^{2}$ distribution), we choose the laser line corresponding to the minimum value of $d_{m}$ as the candidate laser line to be used in the Kalman filter as a measurement. The transformed vision line data also be used in the Kalman filter as the second sensor measurement.

\section{Simulation Results}

We have generated vehicle speed, yaw, laser and vision data in the world coordinate system and simulated with both algorithms. In the simulation, we have given a vehicle path and its corresponding laser and vision data in Figure 3.

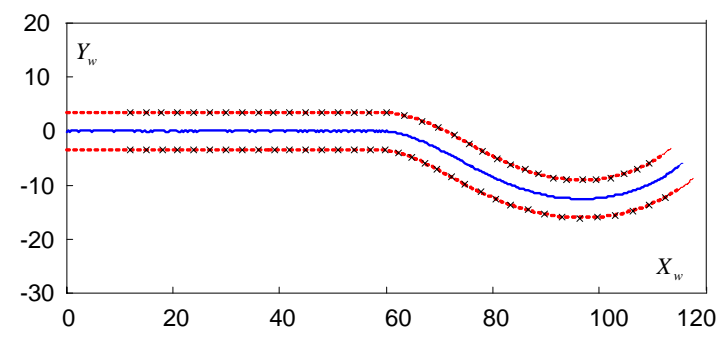

(a) Vehicle path (solid line), laser data (crosses) and actual curbs (dotted lines),

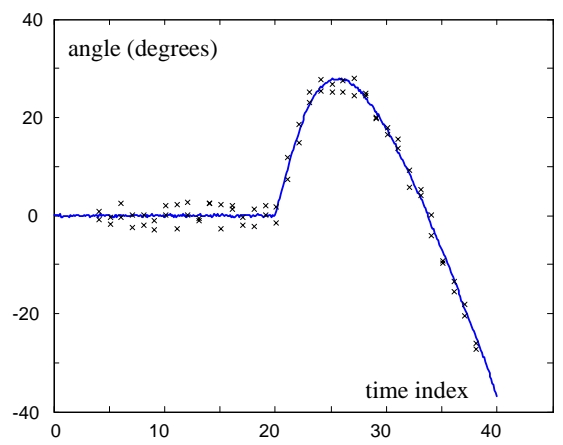

(b) Orientation of actual curbs (solid line) and orientation of corresponding laser measurements (crosses)

Figure 3. Simulated data

The vehicle path consists of a straight-line segment followed by a right and left turn. The tracking performance of both laser alone and image-enhanced algorithms are given in Figure 4. 


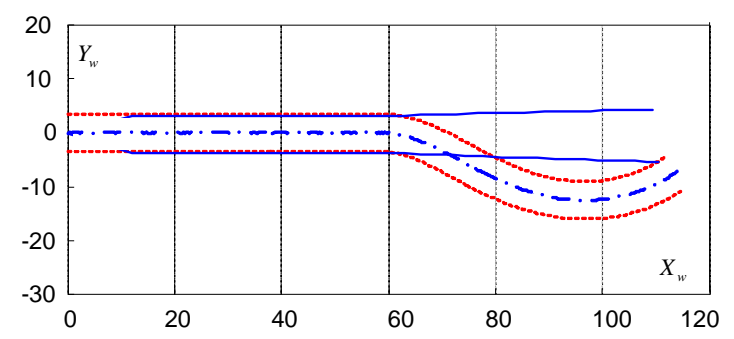

(a) Laser alone tracking (solid), actual (dotted)

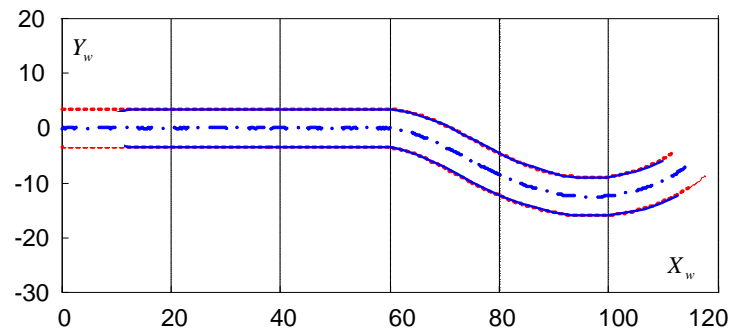

(b) Image-enhanced tracking (solid), actual (dotted)

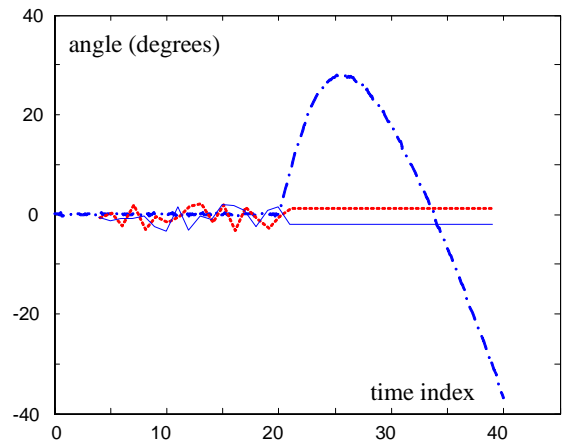

(c) Laser alone orientation tracking (left curb - solid, right curb - dotted), actual (dash dotted)

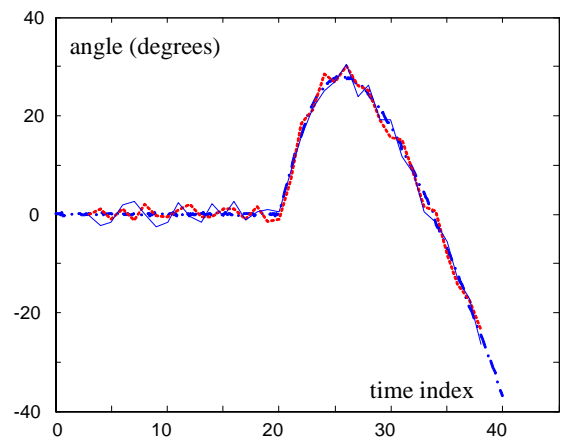

(d) Image-enhanced orientation tracking (left curb - solid, right curb - dotted), actual (dash dotted)

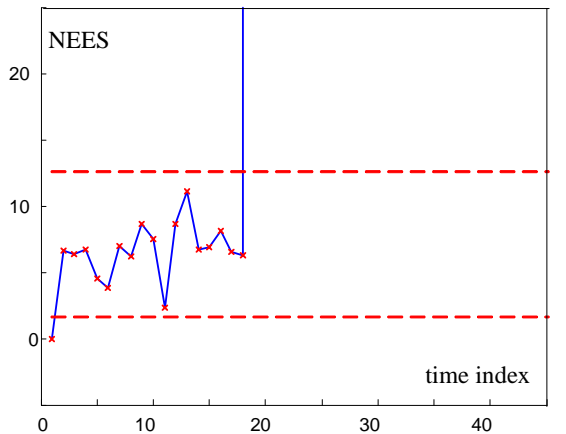

(e) NEES for laser alone

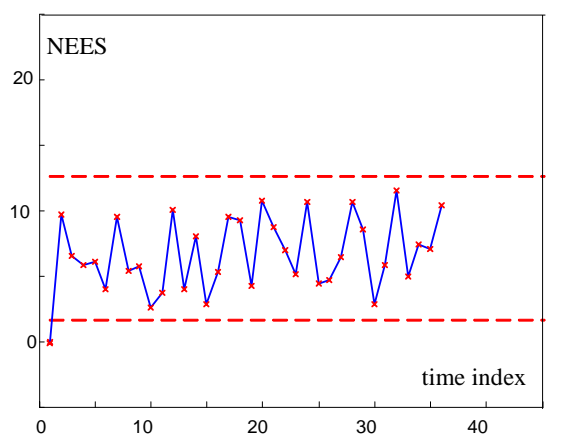

(f) NEES for image-enhanced

Figure 4. Comparison of laser-alone and image-enhanced algorithms

It can be noted that the tracking performance in the straight-line segment is acceptable for both methods, however with the introduction of the bend, the laser alone method fails. It is due to the lack of information about the future bends in the model used. On the other hand, the image-enhanced method uses such road information in the model and hence it is capable of handling bends.

Normalized Estimation Error Squared (NEES): Filter consistency is evaluated using NEES, which is defined as,

$\varepsilon(k)=\tilde{\mathbf{x}}(k / k)^{T} \mathbf{P}(k / k) \tilde{\mathbf{x}}(k / k)$

where, $\tilde{\mathbf{x}}(k / k)={ }^{k} \mathbf{x}(k)-{ }^{k} \hat{\mathbf{x}}(k / k) . \quad \varepsilon(k)$ is chi-square distributed with the acceptance interval, $\varepsilon(k) \in\left[r_{1}, r_{2}\right]$, which is determined such that $P\left\{\varepsilon(k) \in\left[r_{1}, r_{2}\right] \mid H_{o}\right\}=1-\alpha$. The interval $\varepsilon(k) \in\left[r_{1}, r_{2}\right]$ is taken as the $95 \%$ probability region.

It can be seen from Figure 4. (e) and (f) that during the straight line segment both filters are consistent, however once the target starts to maneuver, the laser alone method is far out from the desired region and Chi-square test fails. Image-enhanced tracking method is capable of being consistence throughout the journey.

Another simulation carried out for highly maneuvering road environments with the presence of clutter is shown in 
Figure 5. We only show the performance of imageenhanced technique as the laser alone method can only track during the first straight-line portion.

It can be seen from Figure 5 that the highly maneuvering road scenarios in clutter can also be effectively and consistently handled by the proposed image-enhanced tracking method.

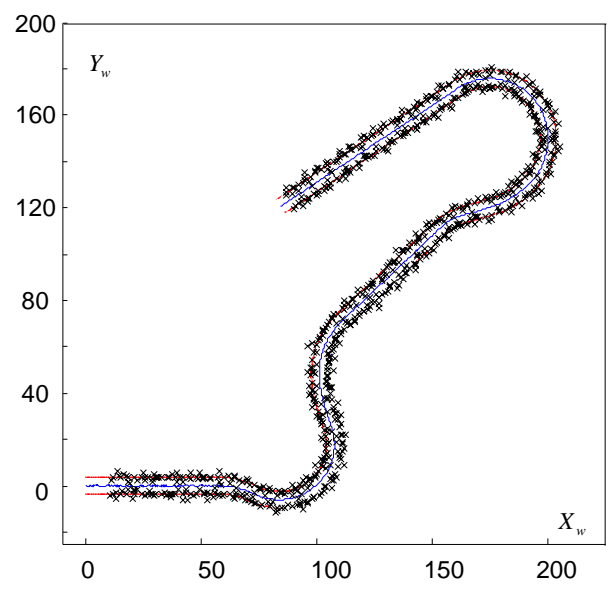

(a) Vehicle path (solid), laser data (crosses)

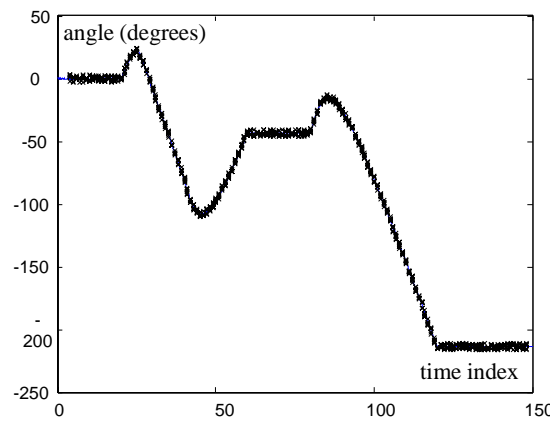

(b) Orientation of actual curbs (solid line) and orientation of corresponding laser measurements (crosses)

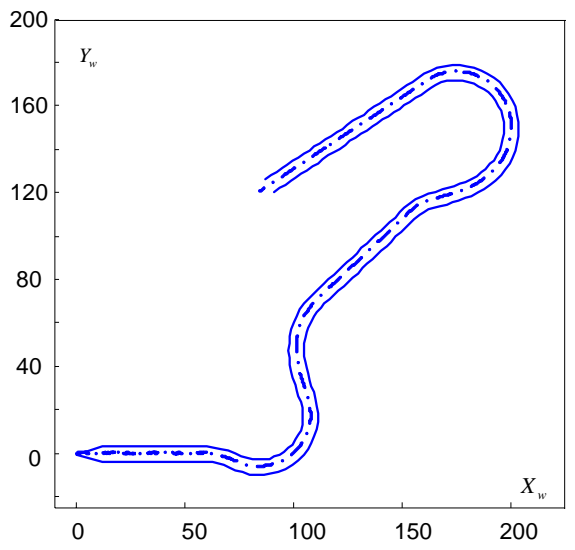

(c) Image-enhanced tracking (solid), actual (dotted)

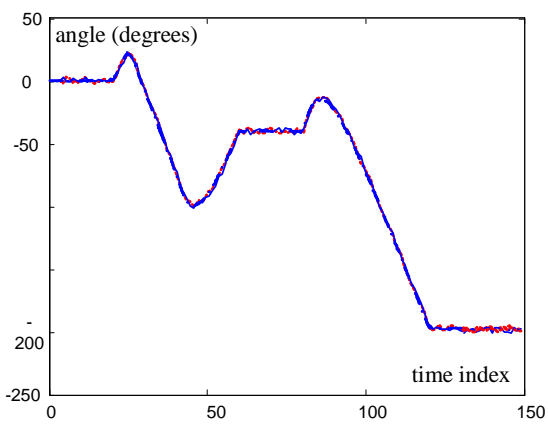

(d) Image-enhanced orientation tracking (left curb solid, right curb - dotted), actual (dash dotted)

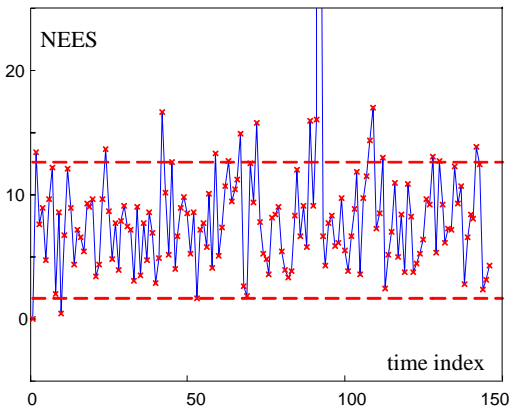

(e) NEES for image-enhanced

Figure 5. Tracking a maneuvering target in clutter

\section{Experimental Results:}

We carried out experiments in campus environments using our GenOME test bed (see Figure 1). Figure 6 shows the map of the test site.

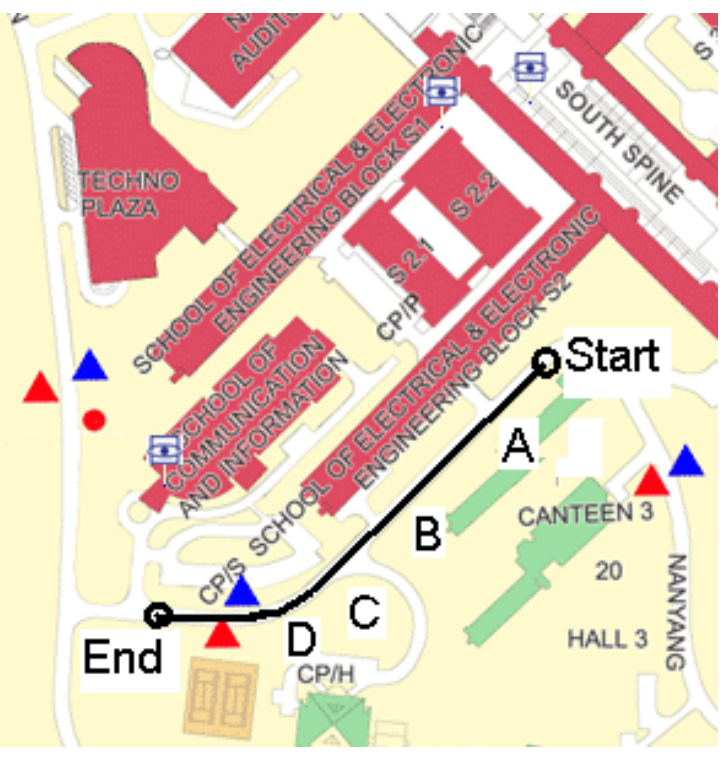

Figure 6. Experimental test site 


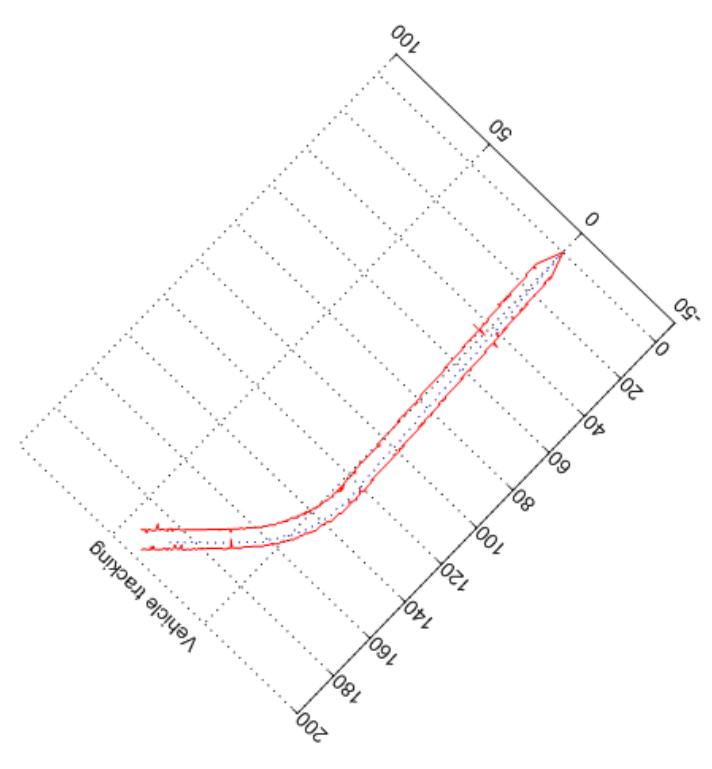

Figure 7. Image enhanced tracking results

In order to facilitate the visualization of the experimental site we have provided some selected images as shown in Figure 8. At position " $\mathrm{A}$ " there is a right road branch and hence there are no curbs present in the right side. In a similar manner position " $\mathrm{B}$ " is having a right road branching and at " $C$ " a left road branching. Position " $D$ ' there is a right turn with a slope. The image-enhanced tracking algorithm proposed is capable of handling such road scenarios with actual experimental data and hence it is a robust method.

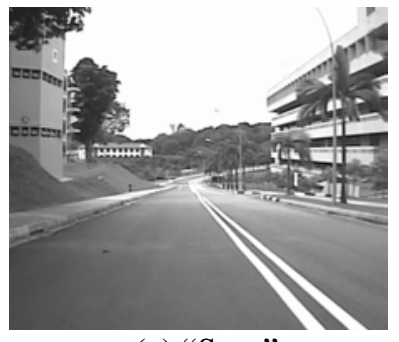

(a) "Start"

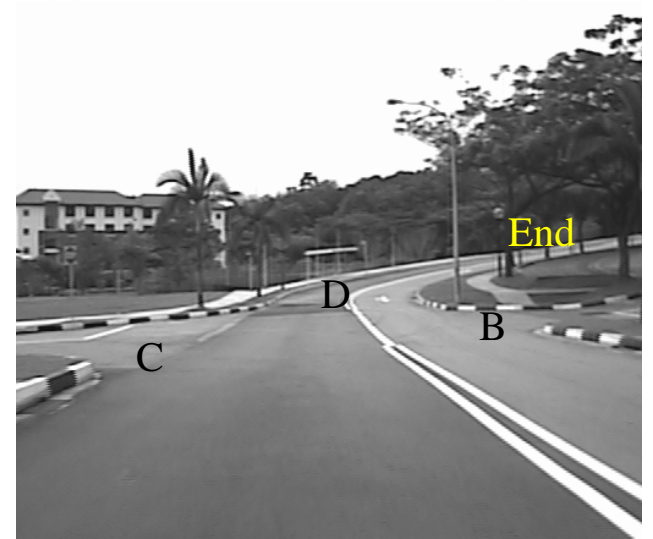

(c) "B,C and D"

Figure 8 Images to show the test site

\section{Conclusions}

In this paper we have investigated handling maneuvering targets in clutter when image-based information are available in addition to 2D LMS data. We have used image-based observations in data association as it correctly reflects the future trends of the road. We also used laser measurements as well as image measurements in our Kalman filter to make it more robust.

We compared the laser-alone tracking method with image-enhanced tracking method and concluded that the image-enhanced tracking method is capable of tracking maneuvering targets in clutter. In contrast, the laser-alone tracking method is only capable of tracking straight-line road segments reliably. Although, there are methods of handling maneuvering targets in clutter only using laser data, the complexity and the accuracy can be improved using additional image data. We have also done experimentations using real data from 2D LMS, CCD camera, gyroscope and wheel encoder data. One of the important observations to make is, the localization errors due to the encoder accumulation error and gyroscope bias error are implicitly handled by the algorithm as it only considers two consecutive vehicle coordinate frames rather than a global coordinate frame.

This algorithm is capable of working amidst roadway obstacles, unless the mid-line is obstructed. Obstructing the mid-line is a problem in the image feature extraction algorithm, however in such cases, we can effectively utilize the previous image data.

\section{References}

[1] D. Pomerleau and T. Jochem, "Rapidly Adapting Machine Vision for Autonomated Vehicle Steering", IEEE Expert, Vol.11 Issue: 2 , pp.19 -27, April 1996.

[2] E. D. Dickmanns and B. D. Mysliwetz, "Recursive 3D Road and Relative Ego-State Recognition", IEEE Transactions of Pattern Analysis and Machine Intelligence, Vol.14, No.2, pp.199-213, 1992.

[3] K. Kluge, "Extracting Road Curvature and Orientation from Image Edge Pointss without Perceptual Grouping into Features", Proceedings of the Intelligent Vehicles'94 Symposium, pp. 109-114, 1994.

[4] S. Lakshmanan and D. Grimmer, "A Deformable Template Approach to Detecting Straight Edges in Radar Images", IEEE Transactions on Pattern Analysis and Machine Intelligence, Vol.18, No.4, pp.438-443, 1996.

[5] K. Kaliyaperumal, S. Lakshmanan and K. Kluge, "An Algorithm for Detecting Roads and Obstacles in Radar Images", IEEE Transactions on Vehicular Technology, Vol.50, No.1,pp.170-182, January 2001. 
[6] M. Nikolova and A. Hero, "Segmentation of a Road from a Vehicle-Mounted Radar and Accuracy of the Estimation", Proceedings of the IEEE Intelligent Vehicles Symposium, pp. 284-289, 2000.

[7] J. Sparbert, K. Dietmayer and D. Streller, "Lane Detection and Street Type Classification using Laser Range Images", Proceedings of IEEE Intelligent Transportation Systems Conference, pp.454-459, Aug. 2001.

[8] Kirchner A. and Heinrich T, "Model Based Detection of Road Boundaries with a Laser Scanner", Proceedings of the International Conference on Intelligent Vehicles, pp.93-98, 1998.

[9] B. Ma, S. Lakshmanan and A. O. Hero, "Simultaneous Detection of Lane and Pavement Boundaries Using Model-Based Multisensor Fusion", The IEEE Transactions on Intelligent Transportation Systems, Vol.1, No.3, pp.135-147, September 2000.

[10] Bar-Shalom Y and Li X. R., Estimation and Tracking: Principles, Techniques and Software, Artech House, Boston.

[11] K.R.S. Kodagoda, W.S. Wijesoma and A.P. Balasuriya, Road Curb and Intersection Detection using a 2D LMS, Proceedings of the IEEE/RSJ International Conference on Intelligent Robots and Systems (IROS 2002), Switzerland, pp.19-24.

[12] W.S.Wijesoma, K.R.S. Kodagoda and A.P. Balasuriya, A Laser-Vision Sensing for Road Detection and Reconstruction, Proceedings of the IEEE $5^{\text {th }}$ International Conference on Intelligent Transportation Systems (ITSC 2002), Singapore, pp.248-253. 